\title{
Triglyceride and Glucose Index (TyG) is a Reliable Biomarker to Predict Non-Alcoholic Fatty Liver Disease
}

\author{
Sania Hossain ${ }^{*}$, Suraya Sultana2 ${ }^{2}$, Khan Md. Shahariar Zaman ${ }^{3}$, Sabrina Shafiq ${ }^{3}$, \\ A. K. M. Shahidur Rahman ${ }^{4}$, Shah Md. Zakir Hossain ${ }^{4}$, Saiful Islam5 ${ }^{5}$, Mahammod Selim Uddin6, \\ Md. Omar Faroque ${ }^{4}$, Sheuly Ferdoushi3 ${ }^{3}$, Debatosh Paul ${ }^{3}$, Md. Quddusur Rahman ${ }^{3}$
}

\footnotetext{
${ }^{1}$ Department of Cytopathology, National Institute of Cancer Research and Hospital (NICRH), Dhaka, Bangladesh

${ }^{2}$ Laboratory Services Division, Sheikh Hasina National Institute of Burn and Plastic Surgery, Dhaka, Bangladesh

${ }^{3}$ Department of Laboratory Medicine, Bangabandhu Sheikh Mujib Medical University (BSMMU), Dhaka, Bangladesh

${ }^{4}$ Department of Nephrology, Bangabandhu Sheikh Mujib Medical University (BSMMU), Dhaka, Bangladesh

${ }^{5}$ Department of Hepatology, Bangabandhu Sheikh Mujib Medical University (BSMMU), Dhaka, Bangladesh

${ }^{6}$ Department of Medicine, Mymensingh Medical College Hospital (MMCH), Mymensingh, Bangladesh

Email: *saniahossaindisha@gmail.com
}

How to cite this paper: Hossain, S., Sultana, S., Zaman, K.Md.S., Shafiq, S., Rahman, A.K.M.S., Hossain, S.Md.Z., Islam, S., Uddin, M.S., Faroque, Md.O., Ferdoushi, S., Paul, D. and Rahman, Md.Q. (2020) Triglyceride and Glucose Index (TyG) Is a Reliable Biomarker to Predict Non-Alcoholic Fatty Liver Disease. Journal of Biosciences and Medicines, 8, 124-136. https://doi.org/10.4236/jbm.2020.811012

Received: October 10, 2020

Accepted: November 15, 2020

Published: November 18, 2020

Copyright $\odot 2020$ by author(s) and Scientific Research Publishing Inc. This work is licensed under the Creative Commons Attribution International License (CC BY 4.0).

http://creativecommons.org/licenses/by/4.0/

(c) (i) Open Access

\begin{abstract}
Non-alcoholic fatty liver disease (NAFLD) is a common liver disease worldwide. There is no specific biomarker for the diagnosis of NAFLD. Triglyceride and glucose index (TyG) may predict the subsequent occurrence of NAFLD in later life. This cross sectional study was aimed to evaluate the effectiveness of triglyceride and glucose index (TyG) as a possible biomarker of NAFLD. The study was conducted at the Department of Laboratory Medicine, Bangabandhu Sheikh Mujib Medical University (BSMMU), Dhaka, Bangladesh from March 2019 to February 2020. A total of 124 subjects were taken as study population following selection criteria. Among them 62 were diagnosed patients of NAFLD and 62 were healthy subjects as control group. Fasting plasma glucose was measured by glucose oxidase method and serum triglyceride was measured by enzymatic-colorimetric method, while TyG index was calculated accordingly. The mean age was $39.5 \pm 11.27$ years in NAFLD patients and $37.10 \pm 12.28$ years in control subjects with male female ratio 1:1.7 and 1:1.8 respectively. Major portion of NAFLD patients (62.9\%) were overweight $(\mathrm{BMI} \geq 25)$. The mean fasting plasma glucose level was $5.73 \pm 1.47$ $\mathrm{mmol} / \mathrm{L}$ in NAFLD patients and $5.27 \pm 0.69 \mathrm{mmol} / \mathrm{L}$ in control group $(\mathrm{p}<$ 0.027). The mean serum triglyceride level was $237.19 \pm 96.47 \mathrm{mg} / \mathrm{dl}$ in NAFLD patients and $117.32 \pm 53.07 \mathrm{mg} / \mathrm{dl}$ in control group $(\mathrm{p}<0.001)$. The triglyceride and glucose index (TyG) was $9.36 \pm 0.47$ in NAFLD group and $8.53 \pm$ 0.42 in control group. TyG index was significantly higher in NAFLD patients
\end{abstract}


in comparison to control group ( $\mathrm{p}<0.001)$. In ROC analysis, cut off value of TyG index was 8.85 with sensitivity $93.5 \%$ and specificity $79 \%$. As a fast and effective method, TyG index can be used as a diagnostic tool to predict NAFLD.

\section{Keywords}

Non-Alcoholic Fatty Liver Disease (NAFLD), Plasma Glucose, Serum

Triglyceride, Triglyceride and Glucose Index (TyG)

\section{Introduction}

Non-alcoholic fatty liver disease (NAFLD) is considered to be a major health problem worldwide due to its increased prevalence [1]. It is a part of the metabolic syndrome characterized by diabetes or pre-diabetes (insulin resistance), being overweight or obese, elevated lipid such as cholesterol and triglyceride with high blood pressure [1]. NAFLD may be defined as steatosis affecting $>5 \%$ of hepatocytes in absence of excessive significant alcohol consumption, other liver disease or the consumption of steatogenic drugs [2]. Histologically NAFLD is divided into nonalcoholic fatty liver (NAFL) or simple steatosis and nonalcoholic steato-hepatitis (NASH) [2].

NAFLD is affecting about one fourth of world population [1]. The prevalence of NAFLD is increasing in Asia pacific region, affecting 30\% of the general population [3]. The prevalence of NAFLD in Bangladesh is $33.86 \%$ and there is no significant difference between the genders [4]. NAFLD is the hepatic manifestation of metabolic syndrome [5]. Hepatic steatosis or triglyceride accumulation is the hallmark histologic feature of NAFLD [6]. Triglyceride accumulation in the hepatocytes is due to an imbalance between lipid accumulation and removal [6]. Several causes may lead to this excess triglyceride accumulation such as increased fat supply due to high fat diet, decreased fat exports in the form of very low density lipoprotein (VLDL) and triglyceride, decreased free fatty acid beta oxidation and de novo lipogenesis [7]. This excess triglyceride leads to development of NAFLD through liver dysfunction [6] [7]. On the other hand, hepatic fat accumulation is related to insulin resistance [8]. Excess fatty acids derived from lipogenesis and fatty acid synthesis; accumulate in peripheral tissues, liver and adipose tissues, resulting in peripheral insulin resistance [9]. It is one of the characteristics features of NAFLD. In the pathogenesis of NAFLD insulin resistance not only increases circulating insulin but also increases hepatic gluconeogenesis, impaired glucose uptake, leading to hyperglycemia [7]. This condition promotes liver fat accumulation and progression of hepatic steatosis. Therefore, when fatty liver develops these two factors, triglyceride and glucose are over produced by the fatty liver [10].

NAFLD is a serious condition and measure should be taken for diagnosis of it as early as possible. There are many invasive and noninvasive markers for the 
diagnosis of NAFLD. To date histopathology of liver tissue is gold standard and reliable for diagnosis of NAFLD [11]. Liver biopsy, though useful, is an invasive procedure with its own complications. Many non-invasive markers are available as an alternative of liver biopsy like-red cell distribution width (RDW), mean platelate volume (MPV), liver function tests such as measurement of aminotransferase level, markers of extracellular matrix remodeling, radiological imaging studies etc [11] [12] [13]. But previous study showed that 79\% of the NAFLD patients had normal ALT [10]. Imaging techniques include ultrasound, computed tomography (CT) and magnetic resonance imaging (MRI) are used to diagnose NAFLD. The sensitivity and specificity of ultrasound of hepatic steatosis are $60 \%$ to $94 \%$ and $66 \%$ to $97 \%$ respectively [14]. But these imaging techniques have some limitations. Computed tomography (CT) has ionizing radiation. Magnetic resonance imaging (MRI) needs expert opinion. Moreover, computed tomography (CT) and magnetic resonance imaging (MRI) are expensive technique. Therefore, it is necessary to find out an easy, reliable and cost effective marker for the diagnosis of NAFLD.

As triglyceride accumulation and insulin resistance is the hallmark of NAFLD, therefore triglyceride and glucose index (TyG) may predict the subsequent occurrence of NAFLD in later life. Studies showed that there is a strong and positive association between TyG and risk of NAFLD in different population [10] [15] [16] [17]. But there is scarce evidence to evaluate the triglyceride and glucose index (TyG) among NAFLD patients in Bangladesh. Therefore, this study aimed to evaluate the role of triglyceride and glucose index (TyG) to identify NAFLD among patients attending a tertiary care hospital (BSMMU) in Bangladesh.

\section{Methodology}

\subsection{Study Design and Study Subjects}

This was a cross sectional study conducted from March 2019 to February 2020 in the Department of Laboratory Medicine, Bangabandhu Sheikh Mujib Medical University (BSMMU), Dhaka, Bangladesh. The study was approved by the Ethical Review Committee, BSMMU, Dhaka, Bangladesh (IRB approval N0. BSMMU/ 2019/8124). According to the statistical calculation, a total of one hundred twenty-four (124) study subjects, age 18 years or above of either sex, who attended the outpatient department of Hepatology, BSMMU, Dhaka, Bangladesh during the study period were included in this study. Among them sixty-two (62) diagnosed patient of NAFLD who fulfilled the selection criteria were selected as cases. At the same time age and sex matched sixty-two (62) healthy subjects as control group were taken. Both NAFLD and control groups were selected according to the ultrasographic evidences.

The exclusion criteria were-pregnant women or lactating mother, subjects having significant alcohol consumption (more than $30 \mathrm{gm} /$ day in case of male and more than $20 \mathrm{gm} /$ day in case of female), subjects carrying hepatitis B \& hepatitis $\mathrm{C}$ virus infections, patients with autoimmune hepatic disease, other chronic 
hepatic diseases i.e. Wilson's disease, hereditary hemochromatosis, primary billiary cirrhosis, patients with diabetes mellitus, hypertension and dyslipidaemia or any other comorbidity and subjects on any medications including vitamin supplementation.

Full explanation regarding the aims, objectives of the study and necessity of the investigations were given to all participants. Informed written consent from the respondents was obtained prior to the enrollment in the study.

\subsection{Sample Size Estimation}

Sample size calculation based on sensitivity (Buderer's formula):

$$
\text { Sample size }(\mathrm{n}) \text { based on sensitivity }=\frac{Z_{1-\alpha / 2}^{2} \times S_{N} \times\left(1-S_{N}\right)}{L^{2} \times \text { Prevalence }}
$$

We hypothesized that TyG index should detect NAFLD with a sensitivity $80 \%$ or greater. The sample size was calculated for a power level greater than $80 \%, \alpha$ error 0.05 and an expected sensitivity $72.2 \%$ based on previous report [10].

The ingredients for the sample size calculation were as follows:

$n=$ required sample size.

$S_{N}=$ anticipated sensitivity.

$\alpha=$ size of the critical region ( $1-\alpha$ is the confidence level).

$Z_{1-\alpha / 2}=$ standard normal deviate corresponding to the specified size of the critical region $(\alpha)$.

$L=$ absolute precision desired on either side (half-width of the confidence interval) of sensitivity.

Prevalence $=$ the estimated prevalence of the disease in the target population (Patients who have clinically suspected nonalcoholic fatty liver disease).

$$
\begin{gathered}
S_{N}=80 \% \\
\alpha=5 \% \\
Z_{1-\alpha / 2}=1.96 \\
L=10 \%
\end{gathered}
$$

Prevalence $=50 \%$ (we consider this value as there was no relevant data in our hospital).

Using Above formula,

$$
\begin{gathered}
n=\frac{1.96^{2} \times 80 \times(1-80)}{0.1^{2} \times 50} \\
\mathrm{n}=123
\end{gathered}
$$

Therefore, final sample size was 124, divided into two groups, NAFLD group $(\mathrm{n}=62)$ and control group $(\mathrm{n}=62)$.

\subsection{Clinical Measurements and Biochemical Analysis}

Each study participants' data-which includes demographic characteristics, medical history, family history, smoking and drinking status, clinical findings and 
laboratory reports were recorded accordingly in a data collection sheet. Their relevant physical examination was done and anthropometry obtained comprised of weight and height. Body mass index (BMI) was calculated as weight (in kilograms)/height square (in meters). BMI was classified according to the World Health Organization (WHO) Asia-Pacific guidelines [18]: normal weight (BMI < $\left.25 \mathrm{~kg} / \mathrm{m}^{2}\right)$ and overweight $\left(\mathrm{BMI} \geq 25 \mathrm{~kg} / \mathrm{m}^{2}\right)$.

Department of Laboratory Medicine, BSMMU was used for estimation of fasting serum triglyceride and fasting plasma glucose. Samples were obtained after an overnight ( $8-12$ hours) fasting. About $4.0 \mathrm{ml}$ venous blood was collected in a plain tube $(6 \mathrm{cc})$ from each participant following standard procedure. Each tube was labeled with the subject's identification number. Then all blood samples were kept at room temperature $\left(22^{\circ} \mathrm{C}-24^{\circ} \mathrm{C}\right)$ in vertical position for 30 minutes. Serum was separated by centrifugation (around $5000 \mathrm{rpm}$ ) for $5-10$ minutes in room temperature and preserved at $-20^{\circ} \mathrm{C}$ until further analysis. Fasting serum triglyceride was measured by enzymatic-colorimetric method and fasting plasma glucose was analyzed by glucose oxidase method. All the measurements were done by an auto-analyzer (SIEMENS Dimension EXL with LM). The product of triglyceride and glucose [triglyceride and glucose index (TyG)] was calculated by recognized formula [19].

\subsection{Estimation of Triglyceride and Glucose Index (TyG)}

The triglyceride and glucose index (TyG) of each study subject's was calculated by the following formula [19]:

$$
\text { TyG }=\operatorname{Ln}[\text { fasting triglyceride }(\mathrm{mg} / \mathrm{dl}) \times \text { fasting glucose }(\mathrm{mg} / \mathrm{dl}) / 2]
$$

\subsection{Statistical Analysis}

Data cleaning validation and analysis was performed using the SPSS (Statistical Package for Social Science version 26.0) software. Categorical data were presented as frequency/percentage and continuous variable was expressed as mean \pm SD (standard deviation). Chi-square test, Unpaired Student " $t$ " test and Receiver-operator characteristic (ROC) curve were used to analysis data. $\mathrm{p}$ value $<$ 0.05 was considered as a level of significance.

\section{Results}

This study was intended to evaluate the role of triglyceride and glucose index (TyG) to identify patients with non alcoholic fatty liver disease (NAFLD) in Bangladeshi adults. Total 124 study subjects were included in this study. Among them 62 were NAFLD patients and 62 were healthy subjects as control group. The mean age was $39.5 \pm 11.27$ years (range $20-70$ years) in NAFLD group and 37.10 \pm 12.28 years (range $20-60$ years) in control group, difference of mean age between the groups was not statistically significant $(\mathrm{p}=0.258)$. The majority $(33.9 \%)$ of the NFALD patients were in $31-40$ years age group, while the majority $(40.3 \%)$ of the control subjects were between the 20 - 30 years age group (Table 1 ). 
Among total study population female were more frequent in both group with male female ratio was 1:1.7 and 1:1.8 respectively, although the gender differences between two groups were not statistically significant $(\mathrm{p}=0.852)($ Table 2$)$.

Majority of NAFLD patients (62.9\%) were overweight (BMI $\left.\geq 25 \mathrm{~kg} / \mathrm{m}^{2}\right)$ and a large percentage of control subjects $(74.2 \%)$ were within normal body weight $\left(B M I<25 \mathrm{~kg} / \mathrm{m}^{2}\right)$. There was significant difference in BMI between NAFLD group and control group $(\mathrm{p}<0.001)$ (Figure 1$)$.

It was observed that mean fasting plasma glucose level was $5.73 \pm 1.47 \mathrm{mmol} / \mathrm{L}$

Table 1. Distribution of age of the study subjects between NAFLD group and control group $(\mathrm{N}=124)$.

\begin{tabular}{cccc}
\hline Age (in years) & $\begin{array}{c}\text { NAFLD group }(\mathrm{n}=62) \\
\text { No. (\%) }\end{array}$ & $\begin{array}{c}\text { Control group }(\mathrm{n}=62) \\
\text { No. }(\%)\end{array}$ & p value \\
\hline $20-30$ & $16(25.8)$ & $25(40.3)$ & \\
$31-40$ & $21(33.9)$ & $17(27.4)$ & \\
$41-50$ & $15(24.2)$ & $12(19.4)$ & \\
$51-60$ & $8(12.9)$ & $8(12.9)$ & \\
$61-70$ & $2(3.2)$ & $0(0.0 \%)$ & $0.258^{\text {ns }}$ \\
Mean \pm SD & $39.50 \pm 11.27$ & $37.10 \pm 12.28$ & \\
Range & $20-70$ & $20-60$ & \\
\hline
\end{tabular}

Unpaired student t-test was performed to compare between two groups; Data were expressed as frequency with percentage and mean $\pm \mathrm{SD}$; Values in the parentheses denote the corresponding percentage; ns = not significant.

Table 2. Gender distribution of the study subjects between NAFLD group and control group $(\mathrm{N}=124)$.

\begin{tabular}{cccc}
\hline Gender & $\begin{array}{c}\text { NAFLD group } \mathbf{n}=62 \\
\text { No. (\%) }\end{array}$ & $\begin{array}{c}\text { Control group } \mathbf{n}=62 \\
\text { No. (\%) }\end{array}$ & p value \\
\hline Male & $23(37.1)$ & $22(35.5)$ & $0.852^{\mathrm{ns}}$ \\
Female & $39(62.9)$ & $40(64.5)$ & \\
Male: Female ratio & $1: 1.7$ & $1: 1.8$ \\
\hline
\end{tabular}

Chi-squared Test $\left(\chi^{2}\right)$ was done to analyze the data; Values in the parentheses denote the corresponding percentage; $\mathrm{ns}=$ not significant.

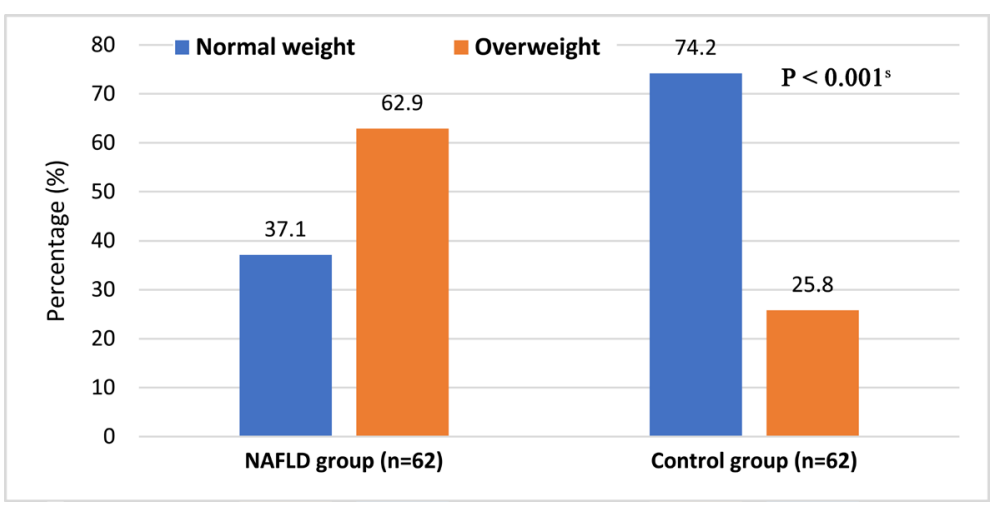

Figure 1. Bar diagram shows BMI distribution of the study subjects $(\mathrm{N}=124)$. 
in NAFLD patients and $5.27 \pm 0.69 \mathrm{mmol} / \mathrm{L}$ in control group. The difference was statistically significant between the groups ( $\mathrm{p}<0.027$ ). The mean serum triglyceride level was $237.19 \pm 96.47 \mathrm{mg} / \mathrm{dl}$ in NAFLD group and $117.32 \pm 53.07 \mathrm{mg} / \mathrm{dl}$ in control group. Serum triglyceride level was significantly higher in NAFLD patients compared to the control subjects $(\mathrm{p}<0.001)$ (Table 3$)$.

The triglyceride and glucose index $(\mathrm{TyG})$ were 8.6 - $10.7(9.36 \pm 0.47)$ in NAFLD group and $7.7-9.3(8.53 \pm 0.42)$ in control group. TyG index was significantly higher in NAFLD patients in comparison to control group $(\mathrm{p}<0.001)$ (Table 4).

Figure 2 showed that the best cut off point of TyG index (blue color) was 8.85 for NAFLD. It showed the highest sensitivity (93.5\%) and specificity (79\%) with an area under curve (AUC) of 0.916 (95\% CI; $0.0868-0.964),(\mathrm{p}<0.001)$.

Table 5 shows ROC curve analysis. It was found that, cut off point of TyG index was 8.85 for NAFLD. That was the highest sensitivity (93.5\%) and specificity $(79 \%)$ with an area under curve (AUC) of 0.916 (95\% CI; $0.0868-0.964)$, (p < 0.001).

Table 6 shows effectiveness of TyG index to predict NAFLD. TyG index shows $93.55 \%$ sensitivity, $79.03 \%$ specificity, $81.69 \%$ positive predictive value and $92.45 \%$ negative predictive value.

Table 3. Comparison of fasting plasma glucose and serum triglyceride between NAFLD group and control group $(\mathrm{N}=124)$.

\begin{tabular}{cccc}
\hline Variables & $\begin{array}{c}\text { NAFLD group }(\mathrm{n}=62) \\
\text { Mean } \pm \text { SD }\end{array}$ & $\begin{array}{c}\text { Control group }(\mathrm{n}=62) \\
\text { Mean } \pm \text { SD }\end{array}$ & p value \\
\hline Fasting plasma glucose & $5.73 \pm 1.47 \mathrm{mmol} / \mathrm{L}$ & $5.27 \pm 0.69 \mathrm{mmol} / \mathrm{L}$ & $0.027^{\mathrm{s}}$ \\
Triglyceride & $237.19 \pm 96.47 \mathrm{mg} / \mathrm{dl}$ & $117.32 \pm 53.07 \mathrm{mg} / \mathrm{dl}$ & $<0.001^{\mathrm{s}}$ \\
\hline
\end{tabular}

Unpaired student t-test was performed to compare between the groups; Data were expressed as mean $\pm \mathrm{SD}$; $s=$ significant.

Table 4. Comparison of triglyceride and glucose index (TyG) between NAFLD group and control group $(\mathrm{N}=124)$.

\begin{tabular}{cccc}
\hline Variables & $\begin{array}{c}\text { NAFLD group }(\mathbf{n}=62) \\
\text { Range }(\text { Mean } \pm \text { SD) }\end{array}$ & $\begin{array}{c}\text { Control group }(\mathbf{n = 6 2}) \\
\text { Range }(\text { Mean } \pm \text { SD) }\end{array}$ & p value \\
\hline TyG index & $8.6-10.7(9.36 \pm 0.47)$ & $7.7-9.3(8.53 \pm 0.42)$ & $<0.001^{\text {s }}$ \\
\hline
\end{tabular}

Unpaired student t-test was performed to compare between two groups; Data were expressed as range $($ mean $\pm \mathrm{SD})$; $\mathrm{s}=$ significant.

Table 5. Receiver-operator characteristic (ROC) curve of TyG index to predict NAFLD (n $=124)$.

Test Result Variable(s): triglyceride and glucose index (TyG)

\begin{tabular}{ccccccc}
\hline \multirow{2}{*}{ AUC } & Cutoff point & p-value & Sensitivity & Specificity & \multicolumn{2}{c}{$95 \%$ CI } \\
\cline { 5 - 6 } & & & & & Lower & Upper \\
\hline 0.916 & 8.85 & $<0.001$ & $93.5 \%$ & $79.03 \%$ & 0.868 & 0.964 \\
\hline
\end{tabular}




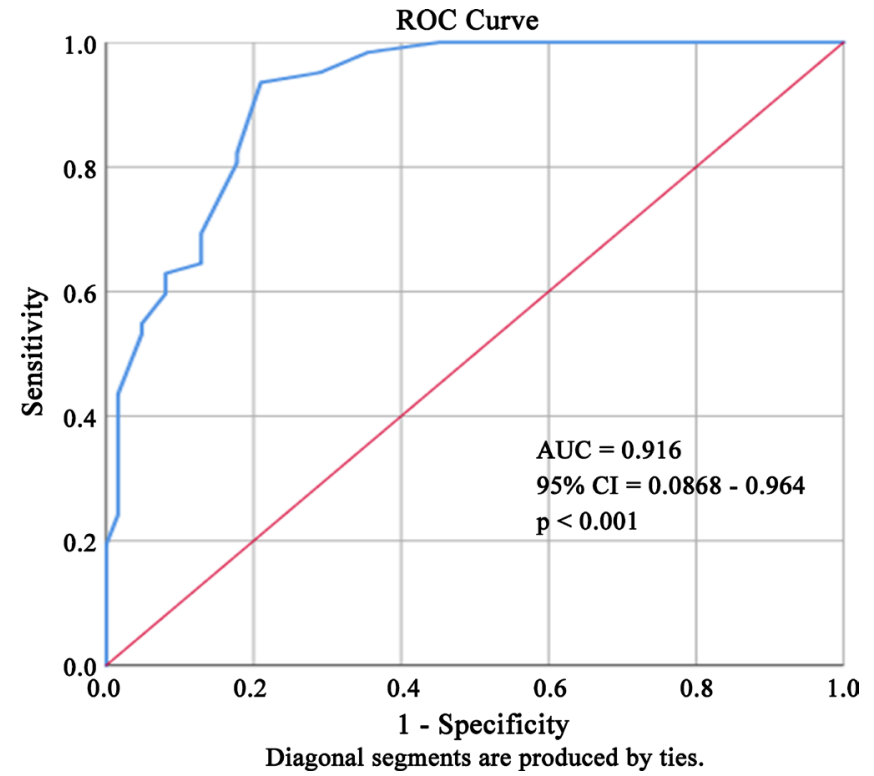

Figure 2. Receiver operating characteristic (ROC) curve of TyG index for the best prediction of NAFLD.

Table 6. Sensitivity and specificity of triglyceride and glucose index (TyG) to predict NAFLD.

\begin{tabular}{cc}
\hline Parameters & Percentage (\%) \\
\hline Sensitivity & $93.55 \%$ \\
Specificity & $79.03 \%$ \\
Positive Predictive Value & $81.69 \%$ \\
Negative Predictive Value & $92.45 \%$ \\
\hline
\end{tabular}

\section{Discussion}

Non alcoholic fatty liver disease (NAFLD) has become one of the major causes of chronic liver disease and recently being an important public health concern worldwide. Several biomarkers associated with NAFLD have been the subject of research in last few decades. Biomarker related with metabolic syndrome provides valuable and timely diagnostic information for NAFLD. NAFLD is not only the hepatic manifestation of metabolic syndrome but also associated with type-2 diabetes mellitus, dyslipidemia, and hypertension [1]. Therefore, there is a possible relationship between triglyceride and glucose index (TyG) with NAFLD. In this study we compared the triglyceride and glucose index (TyG) between NAFLD patients and healthy control subjects. Total 124 subjects (62 diagnosed NAFLD patients and 62 healthy control subjects) were included.

In our study, it was observed that mean age of NAFLD patients was $39( \pm 11.27)$ years and that of control group was $37( \pm 12.28)$ years, the difference of mean age between the groups was not statistically significant $(\mathrm{p}=0.258)$. These finding was consistent with previous studies [15] [20] [21]. While, in a study by Lee et al. the mean age of NAFLD patients was $53.11( \pm 8.58)$ years and in control group 
that was $52.29( \pm 9.70)$ years [5]. The mean age difference between two groups in their study was significant $(\mathrm{p}<0.01)$. This result was not consistent with our study. It may be due to small sample size or demographic variable.

In our study it was observed that female were predominant in both groups. Alam et al. in a similar study showed female predominance, where female was $59.2 \%$ and male was $40.8 \%$ among NAFLD patients [22]. Another related study showed female predominating in NAFLD patients and that was 57\% [23]. The results of the present study closely resembles with findings of these previous studies. On the other hand in another study by Zhang et al. observed significant difference $(\mathrm{p}<0.0001)$ of the gender distribution in both groups; where male was $72.1 \%$, female was $27.9 \%$ in NAFLD patients and in control group male was 56.5.\% and female was $43.5 \%$ respectively [10]. These results were not consistent with this present study. It was reported that, fatty liver disease is a male predominant disease [10] [24], but we found female predominance in our study. This female predominance in this current study may be due to social conservative attitude which bounded most of females to stay at home for household activities, leading to sedentary life style and also due to demographic variable as reported in a previous study [4].

In this present study it was observed that majority of NAFLD patients (62.9\%) were overweight $\left(\mathrm{BMI} \geq 25 \mathrm{~kg} / \mathrm{m}^{2}\right)$ and a large percentage of control subjects (74.2\%) were within normal body weight (BMI $\left.<25 \mathrm{~kg} / \mathrm{m}^{2}\right)$. In our study, the difference of bodyweight between the groups was statistically significant ( $\mathrm{p}<$ 0.001). Similar results were observed in previous studies and reported that BMI is an important factor affected TyG efficacy for identifying the individuals with risk of NAFLD [4] [10] [24].

In this study, fasting plasma glucose and serum triglyceride were measured in NAFLD and control group. The mean fasting plasma glucose level was significantly higher in NAFLD patients than control group $[5.73( \pm 1.47) \mathrm{mmol} / \mathrm{L}$ and 5.27 $( \pm 0.69) \mathrm{mmol} / \mathrm{L}$ respectively, $(\mathrm{p}<0.027)$ ]. Zhang et al. in their study found the mean fasting plasma glucose level in NAFLD patient was $5.20 \mathrm{mmol} / \mathrm{L}$ and in control group that was $4.9 \mathrm{mmol} / \mathrm{L},(\mathrm{p}<0.0001)$ [10]. Similarly, Lee et al. found significant higher fasting plasma glucose level in NAFLD patient $[5.38( \pm 10.19) \mathrm{mg} / \mathrm{dl}]$ compared to control group [5.09 $( \pm 10.50) \mathrm{mg} / \mathrm{dl}],(\mathrm{p}<0.01)$ [5]. Another study by Simental-Mendia et al. reported that the mean plasma glucose level in hepatic steatosis patients was significantly higher compared to control group $[5.7( \pm 1.2)$ $\mathrm{mmol} / \mathrm{L}$ versus $5.3( \pm 2.5 \mathrm{mmol} / \mathrm{L}),(\mathrm{p}<0.001)]$ [25]. On the other hand in this study, there was significant increase in serum triglyceride level in NAFLD patients compared to control group $[237.19( \pm 96.47) \mathrm{mg} / \mathrm{dl}$ versus $117.32( \pm 53.07) \mathrm{mg} / \mathrm{dl}(\mathrm{p}$ $<0.001)$ ]. In a similar study Zhang et al. showed that a significant increase in serum triglyceride level in NAFLD patients $(61.87 \mathrm{mg} / \mathrm{dl})$ when compared to control group $(34.80 \mathrm{mg} / \mathrm{dl})(\mathrm{p}<0.0001)$ [10]. Another related study Simental-Mendia et al. showed that the mean serum triglyceride level was significantly higher in patients with NAFLD $(37.8 \mathrm{mg} / \mathrm{dl})$ in comparison to control group $(19.8 \mathrm{mg} / \mathrm{dl})(\mathrm{p}<0.01)$ 
[25]. These results were also consistent with this present study.

In the current study, it was observed that the mean triglyceride and glucose index $($ TyG) in NAFLD patients was $9.36( \pm 0.47)$ and in control group that was $8.53( \pm 0.42)$. The range of TyG index was 8.6 - 10.7 in NAFLD group and 7.7 9.3 in control group. There was significant increased in TyG index in NAFLD group as compared to control group ( $\mathrm{p}<0.001)$. Zhang et al. have shown that the mean TyG index was 8.8 (ranged 8.4 - 9.3) in NAFLD patients and 8.2 (ranged 7.8 - 8.6) in control group, which was statistically significant ( $\mathrm{p}<0.0001)$ [10]. Another study by Zheng et al. obtained the mean TyG index was 8.58 (ranged 8.25 - 8.91) in NAFLD patients and 8.21 (ranged 7.91 - 8.54) in control group, which was statistically significant $(\mathrm{p}<0.0001)$ [15]. Simental-Mendia et al. showed that the mean TyG index was $4.95( \pm 0.22)$ in NAFLD patients and $4.50( \pm 0.28)$ in control group, which was statistically significant $(p<0.001)$ [25]. The results of the present study closely resemble findings from previous studies.

The receiver-operating characteristics (ROC) curve of TyG index for the diagnosis of NAFLD was depicted in our study. The area under the curve (AUC) for predicting the patients with NAFLD was 0.916 (95\% CI, 0.868 - 0.964). Zhang et al. found the AUC was 0.782 (0.773 - 0.793) for diagnosis of NAFLD [10]. Another study by Zheng et al. found the AUC to predict the development of NAFLD was 0.76 (0.74 - 0.77) [15]. Yu et al. found the AUC of the ROC curve for TyG index for metabolic unhealthy male was 0.863 (95\% CI, 0.857 - 0.869) and in female that was 0.867 (95\% CI, 0.862 - 0.872) [26]. The finding of the present study was supported by the findings of those previous studies.

Using the receiver-operating characteristics (ROC) curve we calculate the potential cut-off value to diagnosis of NAFLD patients. An optimal cut-off value of TyG index for NAFLD patient was found 8.85. In this study, sensitivity and specificity of TyG index was found $93.5 \%$ and $79.03 \%$ respectively. Zhang et al. found cut-off point of TyG for diagnosing NAFLD was 8.5, with sensitivity of $72.2 \%$ and specificity of $70.5 \%$ [10]. Another study by Zheng et al. showed the cut-off point of TyG index for predicting NAFLD was 8.52, with sensitivity $67.3 \%$ and specificity $71.9 \%$ [15]. Simental-Mendia et al. observed the cut-off point of TyG index was 4.58 with sensitivity $94 \%$, specificity $69 \%$ [25]. These findings were nearly consistent with the findings of this current study.

This study demonstrated a strong and positive association between TyG index and risk of NAFLD. Liver biopsy is an invasive procedure and the imaging techniques for NAFLD are not convenient in routine clinical practice, hence serum biomarkers are preferred. Therefore the TyG index may be widely used for identification of individuals with NAFLD. It is concluded that cut-off value for TyG index in our study population was 8.85 , which might help the physicians as an additional tool for diagnosis of NAFLD.

\section{Conclusion}

The occurrence of NAFLD significantly increases with increasing level of TyG 
index. It is a simple biomarker for routine clinical practice which can be done easily. Therefore, measurement of TyG index is effective to identify individuals at risk for NAFLD. A TyG cut-off value of 8.85 was highly sensitive for detecting NAFLD subjects and could be used as a diagnostic tool for NAFLD in Bangladeshi adults.

\section{Limitations}

It was a single centre study with a relatively small sample size. Moreover, the diagnosis of NAFLD was based on ultrasonography findings that may be less accurate compared to the diagnosis based on a liver biopsy.

\section{Recommendation}

A population based prospective study with large sample size should be done to establish the triglyceride and glucose index (TyG) as a reliable biomarker of NAFLD.

\section{Acknowledgements}

The authors greatly acknowledge Department of Laboratory Medicine, BSMMU, Dhaka, Bangladesh for their technical support.

\section{Conflicts of Interest}

The authors declare no conflicts of interest regarding the publication of this paper.

\section{References}

[1] Younossi, Z.M., Koenig, A.B., Abdelatif, D., Fazel, Y., Henry, L. and Wymer, M. (2016) Global Epidemiology of Nonalcoholic Fatty Liver Disease-Meta-Analytic Assessment of Prevalence, Incidence, and Outcomes. Hepatology, 64, 73-84. https://doi.org/10.1002/hep.28431

[2] McPherson, S., Hardy, T., Henderson, E., Burt, A.D., Day, C.P. and Anstee, Q.M. (2015) Evidence of NAFLD Progression from Steatosis to Fibrosing-Steatohepatitis Using Paired Biopsies: Implications for Prognosis and Clinical Management. Journal of Hepatology, 62, 1148-1155. https://doi.org/10.1016/j.jhep.2014.11.034

[3] Alam, S., Hossain, M.M., Azam, G., Mustafa, G., Alam, M., Sayeed, A., Khan, M. and Ahmad, N. (2017) Nonalcoholic Fatty Liver Disease: A New Frontier for Hepatology in Bangladesh and a Call for Action to Combat. Journal of Bangladesh College of Physicians and Surgeons, 35, 184-191. https://doi.org/10.3329/jbcps.v35i4.34739

[4] Alam, S., Fahim, S.M., Chowdhury, M.A., Hassan, M.Z., Azam, G., Mustafa, G., Ahsan, M. and Ahmad, N. (2018) Prevalence and Risk Factors of Non-Alcoholic Fatty Liver Disease in Bangladesh. JGH Open, 2, 39-46. https://doi.org/10.1002/jgh3.12044

[5] Lee, S.B., Kim, M.K., Kang, S., Park, K., Kim, J.H., Baik, S.J., Nam, J.S., Ahn, C.W. and Park, J.S. (2019) Triglyceride Glucose Index Is Superior to the Homeostasis Model Assessment of Insulin Resistance for Predicting Nonalcoholic Fatty Liver Disease in Korean Adults. Endocrinology and Metabolism, 34, 179-186. 
https://doi.org/10.3803/EnM.2019.34.2.179

[6] Kawano, Y. and Cohen, D.E. (2013) Mechanisms of Hepatic Triglyceride Accumulation in Non-Alcoholic Fatty Liver Disease. Journal of Gastroenterology, 48, 434-441. https://doi.org/10.1007/s00535-013-0758-5

[7] Yu, J., Marsh, S., Hu, J., Feng, W. and Wu, C. (2016) The Pathogenesis of Nonalcoholic Fatty Liver Disease: Interplay between Diet, Gut Microbiota, and Genetic Background. Gastroenterology Research and Practice, 2016, Article ID: 2862173. https://doi.org/10.1155/2016/2862173

[8] Edmison, J. and McCullough, A.J. (2007) Pathogenesis of Non-Alcoholic Steatohepatitis: Human Data. Clinics in Liver Disease, 11, 75-104.

https://doi.org/10.1016/j.cld.2007.02.011

[9] Manne, V., Handa, P. and Kowdley, K.V. (2018) Pathophysiology of Nonalcoholic Fatty Liver Disease/Nonalcoholic Steatohepatitis. Clinics in Liver Disease, 22, 23-37. https://doi.org/10.1016/j.cld.2017.08.007

[10] Zhang, S.J., Du, T.T., Zhang, J.H., Lu, H.M., Lin, X., Xie, J.H., Yang, Y. and Yu, X.F. (2017) The Triglyceride and Glucose Index (TyG) Is an Effective Biomarker to Identify Nonalcoholic Fatty Liver Disease. Lipids in Health and Disease, 16, Article No. 15. https://doi.org/10.1186/s12944-017-0409-6

[11] Baršić, N., Lerotić, I., Smirčić-Duvnjak, L., Tomašić, V. and Duvnjak, M. (2012) Overview and Developments in Noninvasive Diagnosis of Nonalcoholic Fatty Liver Disease. World Journal of Gastroenterology, 18, 3945-3954.

https://doi.org/10.3748/wjg.v18.i30.3945

[12] Cengiz, M., Candır, B.A., Yılmaz, G., Akyol, G. and Ozenirler, S. (2013) Is Increased Red Cell Distribution Width an Indicating Marker of Nonalcoholic Steatohepatitis and Fibrotic Stage? World Journal of Gastroenterology, 19, 7412-7418. https://doi.org/10.3748/wjg.v19.i42.7412

[13] Aktas, G., Alcelik, A., Tekce, B.K., Savl, H., Uyeturk, U., Kurt, M., Tekelioglu, V. and Yuce, Y. (2013) Mean Platelet Volume and Red Cell Distribution Width in Hepatosteatosis. National Journal of Medical Research, 3, 264-266.

[14] Machado, M.V. and Cortez-Pinto, H. (2013) Non-Invasive Diagnosis of Non-Alcoholic Fatty Liver Disease. A Critical Appraisal. Journal of Hepatology, 58, $1007-$ 1019. https://doi.org/10.1016/j.jhep.2012.11.021

[15] Zheng, R.J., Du, Z.N., Wang, M.M., Mao, Y.S. and Mao, W.J. (2018) A Longitudinal Epidemiological Study on the Triglyceride and Glucose Index and the Incident Nonalcoholic Fatty Liver Disease. Lipids in Health and Disease, 17, Article No. 262. https://doi.org/10.1186/s12944-018-0913-3

[16] Kim, M.K., Kim, J.H., Park, K., Lee, S.B., Nam, J.S., Kang, S., Park, J.S., Ahn, C.W. and Kim, Y.S. (2018) Relationship between the Triglyceride Glucose Index and the Presence and Fibrosis of Nonalcoholic Fatty Liver Disease in Korean Adults. Diabetes, 67, 612 p. https://doi.org/10.2337/db18-612-P

[17] Inavolu, P., Singla, N., Nunsavata, K. and Bhashyakarla, R.K. (2018) 14. Triglyceride and Glucose Index (TYG) Index as an Screening Biomarker to Identify Nonalcoholic Fatty Liver Disease. Journal of Clinical and Experimental Hepatology, 8, S41. https://doi.org/10.1016/j.jceh.2018.06.344

[18] World Health Organization Expert Consultation (2004) Appropriate Body-Mass Index for Asian Populations and Its Implications for Policy and Intervention Strategies. Lancet, 363, 157-163. https://doi.org/10.1016/S0140-6736(03)15268-3

[19] Simental-Mendía, L.E., Rodríguez-Morán, M. and Guerrero-Romero, F. (2008) The Product of Fasting Glucose and Triglycerides as Surrogate for Identifying Insulin 
Resistance in Apparently Healthy Subjects. Metabolic Syndrome and Related Disorders, 6, 299-304. https://doi.org/10.1089/met.2008.0034

[20] Pagano, G., Pacini, G., Musso, G., Gambino, R., Mecca, F., Depetris, N., Cassader, M., David, E., Cavallo-Perin, P. and Rizzetto, M. (2002) Nonalcoholic Steatohepatitis, Insulin Resistance, and Metabolic Syndrome: Further Evidence for an Etiologic Association. Hepatology, 35, 367-372.

https://doi.org/10.1053/jhep.2002.30690

[21] Hossain, I.A., Shah, M.M., Rahman, M.K. and Ali, L. (2016) Gamma Glutamyl Transferase Is an Independent Determinant for the Association of Insulin Resistance with Nonalcoholic Fatty Liver Disease in Bangladeshi Adults: Association of GGT and HOMA-IR with NAFLD. Diabetes \& Metabolic Syndrome: Clinical Research \& Reviews, 10, S25-S29. https://doi.org/10.1016/j.dsx.2015.09.005

[22] Alam, S., Gupta, U.D., Kabir, J., Noor-E-Alam, S.M., Chowdhury, Z.R. and Alam, A.K. (2016) Transaminases and Gamma Glutamyl Transpeptidase for Detecting Nonalcoholic Steatohepatitis and Fibrosis in Nonalcoholic Fatty Liver Disease. Bangabandhu Sheikh Mujib Medical University Journal, 8, 61-67. https://doi.org/10.3329/bsmmuj.v8i1.28923

[23] Alam, S., Gupta, U.D., Alam, M., Kabir, J., Chowdhury, Z.R. and Alam, A.K. (2014) Clinical, Anthropometric, Biochemical, and Histological Characteristics of Nonobese Nonalcoholic Fatty Liver Disease Patients of Bangladesh. Indian Journal of Gastroenterology, 33, 452-457. https://doi.org/10.1007/s12664-014-0488-5

[24] Kitae, A., Hashimoto, Y., Hamaguchi, M., Obora, A., Kojima, T. and Fukui, M. (2019) The Triglyceride and Glucose Index Is a Predictor of Incident Nonalcoholic Fatty Liver Disease: A Population-Based Cohort Study. Canadian Journal of Gastroenterology and Hepatology, 2019, Article ID: 5121574.

https://doi.org/10.1155/2019/5121574

[25] Simental-Mendía, L.E., Simental-Mendía, E., Rodríguez-Hernández, H, RodríguezMorán, M. and Guerrero-Romero, F. (2017) The Product of Triglycerides and Glucose as Biomarker for Screening Simple Steatosis and NASH in Asymptomatic Women. Annals of Hepatology, 15, 715-720.

[26] Yu, X.W., Wang, L., Zhang, W.C., Ming, J., Jia, A.H., Xu, S.Y., Li, Q.Y. and Ji, Q.H. (2019) Fasting Triglycerides and Glucose Index Is More Suitable for the Identification of Metabolically Unhealthy Individuals in the Chinese Adult Population: A Nationwide Study. Journal of Diabetes Investigation, 10, 1050-1058.

https://doi.org/10.1111/jdi.12975 